\title{
Responsabilidad social desde la microempresa comercial
} Social responsability from the commercial microcompany Responsabilidade social desde a microempresa comercial

\author{
María Consuelo González Pérez* \\ María Lourdes López López
}

\section{RESUMEN}

Se presentan las acciones de responsabilidad social que se practican desde las microempresas, como un compromiso moral y congruente con los objetivos empresariales, demostrando con ello que no son acciones exclusivas para las grandes corporaciones y que de acuerdo a su estructura y capacidad financiera, han venido implementando principios y políticas responsables como un insumo necesario para su desarrollo y posicionamiento en el mercado. La metodología fue cualitativa, se aplicó un instrumento de investigación basado en la escala de Likert al 50\% de los microempresarios dedicados al comercio al por mayor que están inscritos en el Directorio Estadístico Nacional de Unidades Económicas (INEGI, 2009). Esta investigación se realizó en la Región del Évora del estado de Sinaloa, México.

Palabras clave: microempresas, principios, políticas, responsabilidad social.

\section{ABSTRACT}

They present the actions of social responsibility that are practised from the microcompanies, as a moral and coherent commitment with the managerial aims, demonstrating with it that they are not exclusive actions for the big corporations and that in according to its structure and financial capacity they have been implementing principles and responsible policies as an input necessary for its development and positioning in the market. The methodology was qualitative, there was applied an instrument of investigation based on Likert's scale, to 50\% of the microbusinessmen dedicated to the wholesale trade who are inscribed in the Statistical National Directory of Economic Units (INEGI, 2009). This investigation I realize in the Region of the Evora of Sinaloa's state, Mexico.

Keywords: microcompanies, principles, policies, social responsibility.

\section{RESUMO}

Se apresentam as açôes de responsabilidade social, praticadas desde as microempresas como um compromisso moral e congruente com os objetivos empresariais, demonstrando que náo são açóes específicas para as grandes corporaçóes e que de acordo com a sua estrutura e capacidade financeira, foram implementando princípios e políticas responsáveis como um

Mexicana, Doctora en Desarrollo Organizacional, Profesora e investigadora, Facultad de Ciencias Económico, Administrativas y Tecnológicas, Universidad Autónoma de Sinaloa, México. Correspondencia de la autora: mcgp_4@uas.edu.mx, mcgp_4@hotmail.com.

** Mexicana, Doctora en Estudios Fiscales, Profesora, Escuela de Ciencias Económicas y Administrativas, Universidad Autónoma de Sinaloa, México. Correspondencia de la autora: mlourdes50@hotmail.com. 
insumo necessário para o seu desenvolvimento e posicionamento no mercado. A metodologia foi qualitativa, aplicou-se um instrumento de pesquisa com base na escala de Likert a 50\% dos microempresários dedicados ao comércio por atacado, inscritos no Diretório Nacional de Estatística de Unidades Económicas (INEGI, 2009). Esta pesquisa realizou-se na Regiấo do Évora do estado de Sinaloa, México.

Palavras-chave: microempresas, princípios, políticas, Responsabilidade Social.

\section{Introducción}

A partir de la última década del siglo pasado, las organizaciones han manifestado su preocupación por los efectos que pudieran derivarse del quehacer diario que de forma directa o indirecta, modifican los componentes internos y externos de los que depende su crecimiento y permanencia en el mercado. Por tal motivo, a partir de la publicación del Libro Verde (CCE 2001), como una obligación moral, las grandes corporaciones inician la implementación de acciones responsables que mejoren la vida y el desempeño de sus empleados, fomenten las relaciones con sus clientes y contribuyan con el cuidado y la preservación del medio ambiente.

Sin embargo, estudios realizados por Juholin (2003) en Finlandia, Sanborn (2004), Agüero (2002) y Sánchez (2000) en América Latina, sobre la Responsabilidad Social Empresarial (RSE), indican que son las grandes corporaciones las que han venido implementando acciones responsables que les permiten obtener el reconocimiento social a través del distintivo de empresas socialmente responsables. La práctica de acciones de RS, como una obligación moral para las organizaciones, puede representar el ingrediente que haga la diferencia del resto de las empresas, proyectando una imagen ética que sea reconocida por sus empleados, clientes, proveedores, instituciones y sociedad en la que se encuentra inmerso su mercado.

Esta investigación proporciona información respecto a la preocupación y participación de las microempresas en la práctica de acciones responsables en función de su tamaño y estructura, demostrando con ello el compromiso que tienen dichas empresas con la sociedad, trabajadores y sobre todo con el cuidado y la preservación del medio ambiente.

El gobierno mexicano ha identificado la importancia de las pequeñas organizaciones que conforman más del $95 \%$ del total de la industria que integra el motor productivo del país y que contribuye de manera directa con la generación de empleos, el bienestar social y el desarrollo económico de México. Por tal motivo; a finales del año 2002, se decretó la Ley para el Desarrollo de la Competitividad de la Micro, Pequeña y Mediana 
Empresa (LDCMPME) con el objeto de fomentar este sector y acrecentar su participación en los mercados nacionales e internacionales.

Para microempresa no existe una definición única, la definición varía de un país a otro en función, primero de las características del entorno económico, social e incluso cultural; y después, de las especificidades que adopta el propio sector de la microempresa. Andrade (2005) define a la empresa como una entidad formada con un capital social, y que aparte del propio trabajo de su promotor puede contratar a un cierto número de trabajadores. Su propósito lucrativo se traduce en actividades industriales y mercantiles, o la prestación de servicios.

Las microempresas en México, son pequeńas organizaciones legalmente constituidas que se establecen para desarrollar actividades económicas en menor escala y que tienen entre 0 y 10 trabajadores; en su mayoría son concebidas en el seno familiar. La estratificación en base al número de trabajadores, la establece la LDCMPME en su artículo $3^{\circ}$.

\section{Antecedentes}

La Comisión de las Comunidades Europeas (2001), en su "libro verde", explica que la RSE es esencialmente, un concepto con arreglo a que las empresas decidan voluntariamente contribuir al logro de una sociedad mejor y un medio ambiente más limpio. En un momento en el que la Unión Europea intenta determinar sus valores comunes adoptando una carta de los derechos fundamentales, un número creciente de empresas europeas reconocen cada vez más claramente su responsabilidad social y la consideran como parte de su identidad. Esta responsabilidad se expresa frente a los trabajadores y, en general, frente a todos los interlocutores de la empresa que pueden a su vez influir en su éxito.

Numerosos factores impulsan este avance de la RS de las empresas:

- Las nuevas inquietudes y expectativas de los ciudadanos, consumidores, poderes públicos e inversores en el contexto de la mundialización y el cambio industrial a gran escala.

- Los criterios sociales influyen cada vez más en las decisiones de inversión de las personas o las instituciones, tanto en calidad de consumidores como de inversores.

- La preocupación cada vez mayor sobre el deterioro medioambiental provocado por la actividad económica.

- La transparencia de las actividades empresariales propiciada por los medios de comunicación y las modernas tecnologías de información y comunicación. 
A medida que las propias empresas se enfrentan a los retos de un entorno en mutación en el contexto de la mundialización y, en particular, del mercado interior, aumenta su convencimiento de que la RS puede tener un valor económico directo. Aunque la responsabilidad principal de las empresas consiste en generar beneficios, pueden contribuir al mismo tiempo al logro de objetivos sociales y medioambientales, integrando la responsabilidad social como inversión estratégica en el núcleo de su estrategia empresarial, sus instrumentos de gestión y sus actividades.

Así mimo, el Libro Verde señala que se pueden distinguir dos dimensiones en la RSC: la dimensión interna, que está relacionada con los aspectos que afectan a los trabajadores y al contexto que se vive al interior de la empresa, como son los cambios organizativos e innovaciones tecnológicas y la utilización de los recursos naturales en los procesos productivos. La inversión en recursos humanos, necesaria para mantener un equipo de trabajo involucrado en los objetivos empresariales, encaminados a coadyuvar en el bienestar de los trabajadores y sus familias con actuaciones encaminadas a brindar capacitación, seguridad social, prácticas responsables de contrataciones no discriminatorias con personas mayores, capacidades diferentes, madres solteras, mujeres, religiones, entre otras.

La dimensión externa que está conformada por el entorno local, nacional y mundial, relacionado con las actividades que desarrolla la empresa, como proveedores, consumidores, autoridades fiscales, asociaciones empresariales, al igual que con las instituciones que velan por la protección, observancia y aplicación de los derechos humanos así como la lucha contra la corrupción y los códigos de conducta y los problemas ecológicos mundiales. En esta dimensión se busca que las empresas contribuyan al desarrollo de las comunidades que conforman su mercado, para garantizar su permanencia y reconocimiento social.

Tratándose de microempresas que se caracterizan por estar concebidas en el seno familiar; fundadas por los abuelos, continuadas por los padres, y luego por los hijos, y que se han ido especializando en las actividades que desarrollan con la experiencia misma que adquieren, están muy identificados con las necesidades de las comunidades y comprometidos con la sociedad; por lo que la práctica de acciones responsables contribuye a su posicionamiento en el mercado. Se puede decir que una empresa familiar es aquella cuyo control de la dirección está en manos de una o varias familias; o bien, uno o varios miembros de la familia (Mirell, 2014).

\section{Concepto}

La mayoría de las definiciones de la responsabilidad social de las empresas entienden este concepto como la integración voluntaria, por parte de las empresas, de las preocupaciones sociales y medioambientales en sus operaciones comerciales y sus relaciones 
con sus interlocutores. Ser socialmente responsable no significa solamente cumplir plenamente las obligaciones legales, sino ir más allá de su cumplimiento invirtiendo más en el capital humano, el entorno y las relaciones con los interlocutores.

El CEMEFI (2012) define la RSE como el compromiso consciente y congruente de cumplir integralmente con la finalidad de la empresa, tanto en lo interno como en lo externo, considerando las expectativas económicas, sociales y ambientales de todos sus participantes. De esta forma se da por entendido que la responsabilidad empresarial no es algo ajeno o ańadido a la función original de la empresa. Por el contrario, implica cumplir con ella con la conciencia de que esto impactará de forma positiva o negativa, directa o indirectamente, interna o externamente, a grupos y comunidades vinculadas con su operación. Es la capacidad de responder a estos desafíos buscando maximizar los impactos positivos y minimizar los negativos, haciendo mejores negocios al atender estas expectativas.

La evolución del concepto de RS, según lo explica Guzmán (2004), ha abierto camino para generar lo que hoy en día conocemos como RSE, siendo la dimensión social la que inclina la balanza al momento de emitir juicio sobre la calidad de una determinada actividad empresarial. En razón a ello, lo consideramos un recurso estratégico para la sobrevivencia de las organizaciones públicas y privadas, convirtiéndose la práctica de la justicia en una cuestión rentable, siendo la mejor política económica aquella que produce mejores beneficios sociales.

Por otro lado, Núńez (2006) explica que la RSE tiene que ver con una serie de principios de gestión que, sin ser obligatorios, mejoran sosteniblemente la calidad de vida del entorno, las relaciones entre la empresa y sus accionistas, la autoridad, las organizaciones interesadas y todos los que tienen alguna relación o interés en su actividad. Así mismo, Argandońa (2012) la define como ese conjunto de responsabilidades que la empresa asume ante la sociedad, que irán cambiando, como es lógico, porque cambian las circunstancias y la misma sensibilidad de los actores.

Un entendimiento claro de la RSE es necesario antes de evaluar si una empresa particular ha cumplido con sus obligaciones sociales, es decir, de juzgar el desempeño social. El término RS es también usado para representar a las mismas obligaciones, bien sean impuestas por la sociedad, o aquellas asumidas por una organización particular. La RS, usada de esta manera, se refiere a la obligación que las empresas tienen hacia la sociedad. Hasta ahora esto no es controversial, sin embargo existe un debate sobre el contenido que debe dársele al concepto. Una razón de peso por la que no hay consenso sobre la responsabilidad social de la empresa es que no hay un acuerdo general sobre el propósito de los negocios o no han habido legítimas demandas de esto (Abreu y Badii, 2007). 
Por su parte, Correa (2007) indica que en las dos últimas décadas del siglo pasado, es cuando se comienza a debatir más ampliamente el término de RSC, también llamada RSE, y en diferentes campos académicos y empresariales, es aceptado cada vez más. En un sentido más general la RSC puede describirse como la responsabilidad que la empresa tiene o asume frente a la sociedad en general. Otro concepto que ha ganado una amplia aceptación es el de sostenibilidad, el cual surge de la creciente preocupación mundial sobre el ambiente.

La mayoría de las definiciones de la RS de las empresas, entienden este concepto como la integración voluntaria, por parte de las empresas, de las preocupaciones sociales, educativas, de salud y del medio ambiente en sus operaciones comerciales y sus relaciones con sus grupos de interés tanto internos como externos (Dávila y Gómez, 2009).

Del mismo modo, Aragón y Rocha (2009) la definen como un concepto social en construcción, cuyos límites y potencialidades, tanto en términos teóricos como de implicaciones prácticas, están en buena medida aún por definir. En un periodo histórico marcado por la intensidad y continuidad de los cambios en todas las dimensiones de la vida social, el concepto de RSE debe expresar el compromiso de las organizaciones, instituciones y actores diversos que configuran el tejido social, tanto públicos como privados, de que sus actuaciones y las consecuencias que de ellas se derivan y que deben responder al conjunto de la sociedad.

Actualmente el tema de la RSE se discute a nivel internacional y son muchas las iniciativas u organismos internacionales que se han desarrollado para promover una conducta o cultura empresarial que vaya más allá de la simple generación de utilidades, y que incorpore aspectos sociales y ambientales dentro de la estrategia del negocio. Si bien todas las definiciones presentadas son distintas, todas coinciden en la necesidad de promover las buenas prácticas en los negocios a través de asumir, por parte de la empresa, la responsabilidad de los impactos que genera la actividad que se realiza.

Para la Organización Internacional del Trabajo (OIT, 2010), la responsabilidad social en la empresa, es la manera en que las empresas toman en consideración las repercusiones que tienen sus actividades sobre la sociedad, y en la que afirman los principios y valores por los que se rigen, tanto en sus propios métodos y procesos internos como en su relación con los demás actores. La RSE es una iniciativa de carácter voluntario y que sólo depende de la empresa, y se refiere a actividades que se considera rebasan el mero cumplimiento de la ley.

La RSE debe tener como referente el liderazgo estratégico, la cultura organizacional y el mercado, pero haciendo evidente su sensibilidad social y humana como parte de 
su esencia. Si el comportamiento empresarial es socialmente responsable es porque se incide, se permeabiliza y se evidencia en la sociedad una mayor equidad, una distribución de riqueza más justa y un mejoramiento de la calidad de vida de todos los humanos presentes en su entorno social. El hablar de la RSE, es hablar de un tema que se está trabajando en el ámbito público y privado, como eje estratégico que conlleva competitividad, sostenimiento continuo y desarrollo en las organizaciones; sin importar el sector productivo ni la actividad comercial a la que se dedique, y en la actualidad es una necesidad en la gestión empresarial como mecanismo de reconocimiento y posicionamiento.

No responde a una coyuntura ni a un afán de proyectar una imagen de solidez frente a la comunidad; en realidad responde a una filosofía basada en aspectos éticos y morales que les permita mantenerse en prospectiva de lo que exige una actuación programada dentro de un plan estratégico empresarial que abarque los aspectos humanos, sociales y ambientales; que, en lo interno, eleve sus fortalezas, revierta sus debilidades, y, en el externo, aproveche oportunidades y contrarreste amenazas dentro de un marco de respeto a la institucionalidad, asumiendo responsabilidad en la creación de valor y corresponsabilidad en el proceso de integración social; todo ello orientado a garantizar efectos positivos, especialmente en sectores vulnerables de la sociedad.

Ortiz (2011) explica que es sin duda hasta los años 90 en que el concepto de RSE y la utilización de ese nombre comienza a cobrar protagonismo dentro de la sociedad global, esta profundización y tendencia creciente, se debe al impulso de organismos internacionales y grupos sectoriales que han promovido la creación de códigos de conducta, directrices, normas y otros instrumentos para la implementación de la RSE como una estrategia de negocios integral a largo plazo, que busca convertir a la empresa en un actor que promueva el desarrollo sostenible de su localidad, país y región.

Por otro lado, dos organizaciones empresariales que destacaron por su insistencia y pronunciamiento en el tema, son la Confederación Patronal de la República Mexicana (COPARMEX) y las Uniones Sociales de Empresarios de México, A.C. (USEM). Ambas aportaron elementos teórico-prácticos para la fundamentación y desarrollo del concepto mismo.

Delgado (2013) señala que, hoy en día, la RS es un término que comúnmente se asocia a conceptos relacionados con la ecología, la sustentabilidad, o bien con actividades de compromiso con la sociedad, el desarrollo comunitario e incluso la filantropía. Sin embargo, es un hecho que la RSE no está limitada a realizar acciones en favor del medio ambiente o a prestar ayuda a los que más lo necesitan. Si bien es cierto que la distinción por ser una firma socialmente responsable es cada vez más valorada al interior del 
sector empresarial: aún existe entre la población en general una gran confusión sobre su verdadero significado, debido a que integra diferentes temáticas: económicas, sociales, medioambientales y de gestión interna de la organización, considerando además los conceptos y principios de la ética.

De igual forma, Foretica (2014) define a la RSE como el fenómeno voluntario que busca conciliar el crecimiento y la competitividad integrando al mismo tiempo el compromiso con el desarrollo social y la mejora del medio ambiente. La creciente preocupación de la sociedad sobre los problemas de carácter ético, ambiental y social hace que las empresas traten de abordarlos en profundidad, adquiriendo un compromiso permanente con todos los grupos de interés. De acuerdo a lo planteado por el Centro para la Acción de la Responsabilidad Social Empresarial en Guatemala CENTRARSE (2006), la RSE es hacer negocios basados en principios éticos y apegados a la ley. La empresa (no el empresario) tiene un rol ante la sociedad, y sobre todo, ante el entorno en el que opera.

El objetivo principal que persigue la RSE radica en el impacto positivo que estas prácticas generan en los distintos ámbitos con los que una empresa tiene relación, al mismo tiempo que contribuye a la competitividad y sostenibilidad de la empresa, la que puede orientar sus prácticas responsables hacia el interior de la empresa o hacia fuera de la empresa. En cada caso, ya sea interno o externo, existen diferentes públicos interesados respecto de hacia dónde puede focalizar su acción.

\section{Principios y políticas de responsabilidad social}

Este análisis de la RS nos lleva a distinguir principios de RS internos que rigen en la empresa, así como políticas empresariales dirigidas al cumplimiento de un comportamiento ético con la sociedad. En resumen, se podría afirmar que las crecientes expectativas sobre el nuevo papel de las empresas, han girado en torno a su posible contribución en tres líneas: como instancia generadora de equidad social, de sustentabilidad ambiental y como instancia promotora de desarrollo social. La incorporación de acciones responsables no debe analizarse como un costo, por el contrario, debe tomarse como una inversión que será retribuida en beneficios y mejoras constantes, que permitirán el posicionamiento de la microempresa por medio de la competitividad y autosostenibilidad.

\section{Principios de RS}

La Norma ISO 26000 (2010), establece una guía que contiene y explica los siete principios básicos de la RS que están relacionados entre sí, involucrando en ello su contribución con el desarrollo sostenible; estos principios son: 
Principio 1. Rendición de cuentas.

Principio 2. Transparencia.

Principio 3. Comportamiento ético.

Principio 4. Respeto a los intereses de los stakeholders o grupos de interés.

Principio 5. Respeto al principio de legalidad.

Principio 6. Respeto a la norma internacional de comportamiento.

Principio 7. Respeto a los derechos humanos.

También señala y relaciona al medio ambiente, en el que las decisiones y actividades de las empresas frecuentemente generan un impacto, independientemente del lugar en que estas se encuentren establecidas. Los efectos que se generan están íntimamente ligados con el proceso que la empresa realiza en la transformación de las materias e incorporación de insumos que utiliza y que en un momento dado, pueden ocasionar contaminación y desecho de residuos tóxicos que puedan afectar el ambiente y la estabilidad de los seres vivos.

El CEMEFI (2012) señala que los ámbitos de la RSE responden a principios empresariales universales, y es el conocimiento y la profundización continua de esos principios lo que asegura su implementación exitosa. Los principios que guían la responsabilidad social y la ética empresarial son los siguientes:

- Desempeño ético en los negocios.

- Prevención de negocios ilícitos.

- Respeto a la dignidad humana.

La ética empresarial es la base de las relaciones sólidas entre la empresa, sus proveedores, clientes, accionistas y otros públicos; permite la interpretación y solución de controversias de acuerdo a los principios que guían la toma de decisiones, la formación y evaluación del personal, y la forma en que se debe conducir el negocio.

El principio que guía la responsabilidad social en la vinculación de la empresa con la comunidad es:

- Involucrarse como ciudadano corporativo con las comunidades en las que opera.

La empresa percibe e instrumenta el compromiso y la responsabilidad con su comunidad. Identifica y monitorea las expectativas públicas que la comunidad tiene acerca de ella; la innovación y el involucramiento sostenido de miembros, autoridades $\mathrm{u}$ organizaciones de la comunidad que deben estar incluidos en este monitoreo. Con ello se busca la sinergia entre diversos sectores como la empresa, gobierno y organizaciones de la sociedad civil, 
que permitan multiplicar los resultados en la solución de problemas sociales comunes a todos. Se destacará el papel de cada uno de los participantes y los beneficios que recibirán.

Así mismo los principios que guían la responsabilidad social y la calidad de vida en la empresa son:

- Promover y establecer como prioridad la calidad de vida de la comunidad interna.

- Empleo digno.

La gestión del capital humano, enfocada con la visión de una empresa socialmente responsable, crea internamente un ambiente de trabajo favorable, estimulante, seguro, creativo, no discriminatorio y participativo en el que todos sus miembros interactúan a partir de bases justas de integridad y respeto que propician su desarrollo humano y profesional, contribuyendo para que alcancen una mejor calidad de vida.

Y por último, explica que el principio que guía la responsabilidad social en el cuidado y preservación del medio ambiente es:

- El respeto a su entorno, los recursos y el medio ambiente para el presente y las generaciones futuras.

Uno de los grandes retos de la empresa es combinar la generación de riqueza y la preservación del medio ambiente; estas prácticas demuestran cómo la empresa respeta el medio ambiente, promueve la optimización de recursos, prevé la generación de desperdicios y desarrolla procesos de reciclaje o reaprovechamiento de recursos o incorporación de sus productos y procesos a los ciclos naturales. La empresa debe considerar los factores ambientales como un elemento importante en su toma de decisiones, y reflejar claramente su liderazgo alcanzando la máxima calidad en su manejo y en su relación con el entorno. Por otro lado, cuando se aborda y ejerce la responsabilidad social, el objetivo primordial para una empresa es aumentar al máximo su contribución al desarrollo sostenible, incluyendo la salud y el bienestar de la sociedad.

Brown (2010) coincide con los principios de transparencia, gestión, consumo, normatividad, al igual que FUNDARSE (2009) cuando menciona que las empresas deberán tener plenamente establecidos los principios fundamentales que deben observarse al ejercer su actividad empresarial y tener en consideración las opiniones de los demás terceros interesados.

A continuación se describirán los diez principios que se establecen en el Pacto Mundial (2014), mismos que están basados en declaraciones y convenciones universales aplicadas en cuatro áreas: derechos humanos, medio ambiente, estándares laborales y anticorrupción; iniciando con los que se desglosan de los derechos humanos, los cuales se describirán a continuación: 
Principio uno: Las empresas deben apoyar y respetar la protección de los derechos humanos fundamentales, reconocidos internacionalmente, dentro de su ámbito de influencia.

Principio dos: Las empresas deben asegurarse de que sus empresas no sean cómplices en la vulneración de los derechos humanos.

En relación a los estándares o derechos laborales se encuentran los siguientes:

Principio tres: Las empresas deben apoyar la libertad de asociación y el reconocimiento efectivo del derecho a la negociación colectiva.

Principio cuatro: Las empresas deben apoyar la eliminación de toda forma de trabajo forzoso o realizado bajo coacción.

Principio cinco: Las empresas deben apoyar la erradicación del trabajo infantil.

Principio seis: Las empresas deben apoyar la abolición de las prácticas de discriminación en el empleo y la ocupación.

Y por último, los principios de RS que están relacionados de manera directa con el medio ambiente:

Principio siete: Las empresas deberán mantener un enfoque preventivo que favorezca el medio ambiente.

Principio ocho: Las empresas deben fomentar las iniciativas que promuevan una mayor responsabilidad ambiental.

Principio nueve: Las empresas deben favorecer el desarrollo y la difusión de las tecnologías respetuosas con el medio ambiente.

Principio diez: Las empresas deben trabajar contra la corrupción en todas sus formas, incluidas extorsión y soborno.

\section{Políticas de RS}

El libro verde de la Comisión de las Comunidades Europeas (2001), fundamentalmente podemos distinguir dos dimensiones en la RSC: la dimensión interna, que está relacionada con los aspectos que afectan a los trabajadores y al contexto interno empresarial, la inversión en recursos humanos, la salud y la seguridad, la gestión del cambio y las prácticas respetuosas con el medio ambiente, en cuanto a la gestión de los recursos naturales utilizados en la producción; y la dimensión externa, que está relacionada con el entorno local, ya sea a nivel europeo o mundial, a los socios comerciales, proveedores y consumidores, derechos humanos (lucha contra la corrupción, códigos de conducta) y a los problemas ecológicos mundiales. 
Para Segura (2012), la adopción de criterios de RS en la gestión empresarial significa formalizar políticas y sistemas de gestión en los ámbitos económico, social y ambiental, así como la transparencia informativa respecto a los resultados alcanzados; así mismo pretende intensificar la dimensión social, individual y de grupo, haciendo una empresa más a la medida de la persona, pero para poder llevar a buen término esta gestión es necesario integrar la RS a la sociedad. De igual forma, menciona que la primera responsabilidad del emprendedor o del director de una empresa, es ser una persona ética cuyas competencias profesionales deben ser su deber moral y la obtención de resultados. La combinación de estos dos factores debe ser armoniosa, dando como resultado que el carácter humano de la empresa exija ética y el carácter productivo exija eficiencia.

En este punto es de gran importancia mencionar que las políticas laborales impulsan a la empresa como promotora de la equidad social, ya que ayudan a la disminución de las desigualdades sociales y el respeto a los derechos humanos; esto incluye la generación de empleos dignos desde el punto de vista salarial, de seguridad e higiene, así como también a la inclusión de grupos socialmente marginados, a la igualdad de género, y a la incorporación laboral de un porcentaje de personas con capacidades diferentes.

González (2011), respecto a la política medioambiental, indica que la empresa puede influir en el cuidado del medio ambiente, con responsabilidad que va más allá del cumplimiento de la legislación aplicable; menciona también que la empresa debe tener presente aspectos relativos a la gestión de residuos, a las características de los envases y embalajes, el reciclaje de todos sus desechos, el transporte de mercancías y el consumo responsable de energía. Al respecto Moreno y Graterol (2011) explican que aunque las pequeñas empresas tienen menos impacto negativo medioambiental, todas pueden ayudar reduciendo el consumo de energía, los residuos y reciclando materiales de desecho.

Cabe concluir que se puede considerar a la empresa como una instancia que contribuye a la sustentabilidad, y ello conlleva la disminución de los impactos negativos en el ambiente, siempre que practique algunas de las siguientes actividades o políticas de RS, entre las que se encuentran: el uso de nuevas tecnologías, el ahorro de recursos naturales, entre ellos el agua y la energía principalmente, así como la promoción e instrumentación de una cultura ecológica en todos los niveles.

Lo anterior nos lleva a definir la política ambiental como la preocupación y desarrollo de objetivos con fines para mejorar el medio ambiente, conservar los principios naturales de la vida humana y fomentar el desarrollo sostenible, así como también nos da una idea clara de lo importante que es para la empresa implementar este tipo de políticas. La contribución de la empresa tanto a la equidad como a la sustentabili- 
dad ambiental, se relacionan con "el modo de hacer negocios", y por lo mismo toda organización, independientemente de su giro o tamaño, es susceptible de hacerlo.

La política de mercado es indispensable para la empresa, ya que contribuye a conservar el buen nombre de la empresa, para lo que es de suma importancia actuar correctamente en todas sus relaciones laborales. En la práctica, se puede lograr una buena reputación dejando satisfechos a los clientes, liquidando las facturas a tiempo y cumpliendo con todas las prácticas empresariales de forma ética. El buen nombre es crucial para asegurar el éxito en el mercado y ser sostenible en el tiempo, pero además, una forma rápida de mejorar la productividad de la empresa es compartir experiencias con los proveedores, clientes, empresas de ideas afines y organizaciones empresariales locales, además de otros grupos de interés, para poder iniciar la implementación de la RSE en la cadena de valor (Moreno y Graterol, 2011).

Esta reflexión teórica conlleva a reconocer que los principios y políticas de RS representan un insumo necesario para la empresa, ya que contribuyen al logro de los objetivos económicos, aporta beneficios al individuo, a los colaboradores y a la comunidad; la práctica de los principios y políticas éticas, cívicas y la normatividad legal, la capacitación de los empleados, no solo en asuntos relacionados con su desempeño laboral, sino también en aspectos relacionados con el mejoramiento personal, familiar y social; así como el aseguramiento de las condiciones laborales y de salud de las personas, y la consideración de las decisiones en función de cuestiones éticas y ambientales, repercuten en beneficios financieros e incremento de utilidades en el corto y mediano plazo.

\section{Metodología}

Para conocer las acciones responsables que están practicando las microempresas, se realizó un análisis focalizando las pautas en las áreas que mayor impacto tienen en el entorno, en la calidad de vida de los colaboradores y el cuidado del medio ambiente. La metodología es de tipo cualitativo, a partir de la percepción que tiene el sujeto estudiado desde su propio contexto (Monje, 2011). Se inició con una revisión teórica de los principales autores que han escrito sobre RS, principalmente hablan sobre las grandes corporaciones, sin embargo, en esta investigación se pretende involucrar a las microempresas en la práctica de acciones responsables que involucran al personal que labora en la empresa para contribuir con el cuidado de insumos, la utilización de recursos y fomentar las relaciones interpersonales. Así mismo, que la empresa reconozca y respete los derechos humanos y laborales establecidos en las leyes para que la organización se involucre en la práctica de políticas y principios de RS que contribuyan con la sociedad en la que se desarrollan. 


\section{Sujetos de estudio}

La unidad de análisis fueron los microempresarios dedicados al comercio al por mayor de la Región del Évora, comprendida por los municipios de Salvador Alvarado, Mocorito y Angostura en el estado de Sinaloa, México, respecto a la manera en que implementan principios y políticas de Responsabilidad Social.

\section{Diseño de los instrumentos de investigación}

Como instrumento de recolección de datos se utilizaron dos cuestionarios; el primero para hacer un diagnóstico del conocimiento que los microempresarios tienen respecto a la RS, mismo que se elaboró de manera restringida o cerrada, según lo que explica Osorio (2012) es aquel que solicita respuestas breves, específicas y delimitadas. Para formular preguntas cerradas es necesario anticipar las posibles alternativas de respuestas que se piden, las cuales deben ser contestadas con dos alternativas de respuestas: sí o no. Este cuestionario contiene 20 preguntas y se aplicó a 20 microempresarios dedicados al comercio al por mayor ubicados en la Región del Évora.

Se aplicó un segundo cuestionario para recopilar la información de esta investigación, mismo que fue elaborado de acuerdo a la escala de Likert. En este instrumento se plasmaron 40 preguntas que fueron estructuradas en función de los objetivos planteados al inicio de la investigación. Fue aplicado a 103 microempresarios que representan el 50\% del total registrado en los tres municipios que integran la región del Évora (Mocorito, Angostura y Salvador Alvarado) (INEGI, 2009). El análisis de cada una de las respuestas obtenidas en el instrumento sirvió de base para identificar los principios, políticas y ventajas que obtienen al implementar la RS en las microempresas.

Cabe resaltar la importancia de la estructura del instrumento de investigación que fue elaborado de acuerdo a la escala de Likert, que es una de las más utilizadas para medir actitudes, ya que por su confección y aplicación y el nivel de correlación con otras escalas, es una de las más utilizadas. Malave (2007) explica que dentro del campo de las ciencias sociales, han existido diversas modalidades y tipos de escalas, procedimientos y métodos de confiabilidad y validez que describe la importancia y utilización de herramientas técnicas y estadísticas para el abordaje de una o diversas problemáticas sociales a indagar.

Los cuestionarios con escala Likert han demostrado un reconocido rendimiento en investigaciones sociales en lo que se refiere a la medición de actitudes. Tomando en cuenta que la actitud, por su naturaleza subjetiva, no es susceptible de observación directa, ha de inferirse de la conducta manifiesta; en este caso, a través de la expresión verbal de los sujetos de investigación. 
Por su parte, Ávila (2006) menciona que por lo que se refiere a las escalas Likert, se señalan como ventajas que ellas son más sencillas de contestar, requieren menor trabajo y se realizan de modo más rápido, además de necesitar menor número de ítems para su confección. Parece confirmada la mayor fiabilidad del método Likert, incluso con menor número de enunciados. Como desventajas, se identifica que no permiten determinar en cuánto es más favorable un sujeto que otro respecto a la variable medida. Tampoco es posible establecer en aplicaciones posteriores la cantidad de cambio experimentado en los sujetos.

\section{Aplicación del instrumento}

El instrumento fue dirigido a los microempresarios dedicados al comercio al por mayor que tienen entre cero y diez trabajadores registrados y que están inscritos en el Directorio Estadístico Nacional de Unidades Económicas (DENUE), del INEGI (2009), donde se encuentran registradas microempresas de la región de la siguiente manera:

Tabla 1. Microempresas en la región del Évora.

\begin{tabular}{|l|c|}
\hline Angostura & 49 \\
\hline Mocorito & 24 \\
\hline Salvador Alvarado & 133 \\
\hline Total de la Región & 206 \\
\hline
\end{tabular}

Fuente: elaboración propia en base a INEGI (2009).

La aplicación del instrumento para la recolección de la información, se hizo en el domicilio fiscal de las microempresas, a los dueños o gerentes, en visitas programadas mediante citas telefónicas.

\section{Procesamiento de la información}

Por otro lado los datos obtenidos se alimentaron en una tabla del programa excel para conocer las respuestas de cada una de las preguntas del instrumento de investigación. Cada variable se incluye en una pregunta que debe cubrir de manera satisfactoria los requerimientos según los objetivos planteados. Los resultados se obtienen a partir del análisis de la teoría y los hallazgos producto de la investigación en el trabajo de campo, analizando los gráficos de cada pregunta elaborada y relacionada con cada una de las variables planteadas en la investigación. 


\section{Resultados}

Las principales actividades que se desarrollan en la Región del Évora son: comerciales, agrícolas, ganaderas, pesqueras y algunas otras de menor nivel, sin embargo; esta investigación está dirigida al comercio al por mayor que desarrollan sus habitantes como lo son: la compraventa en abarrotes, lubricantes, pinturas, refacciones, ferreterías, tiendas de ropa, zapatos, materiales para construcción, carnicerías, mercerías y papelerías, mercados, entre otras algunas otras.

Las microempresas de la Región del Évora, coinciden en que es necesaria la práctica de acciones de RS desde la microempresa y reconocen que no es exclusivo de las grandes corporaciones llevar a cabo esta práctica, ya que cada empresa de acuerdo a sus condiciones y estructura puede implementar este tipo de acciones en beneficio de la sociedad en la que se desarrollan. Estas empresas tienen un nicho de mercado distinto a las grandes corporaciones y se deben al lugar donde nacieron y permanecen, esto indica que se trata de empresas familiares; por lo que reconocen que deben contribuir con la sociedad que las prefiere en el mercado.

Se pudo constatar que estas pequeñas organizaciones hacen aportaciones en colectas de la Cruz Roja y Bomberos, que son asociaciones civiles que brindan sus servicios a la comunidad en general, sin perseguir un beneficio a cambio. Reconocen que es innegable que las empresas generan desperdicios por sus operaciones; lo importante es conocer lo que están haciendo para contribuir con el cuidado del medio ambiente. Para ello se practica el reciclaje de los desechos, se utiliza papel de reciclaje y se adquiere papel reciclado, por lo que se pudo identificar que un alto número de microempresas se preocupan por contribuir al desarrollo de la comunidad y la protección del medio ambiente.

Se logró identificar en la Región del Évora, a una empresa dedicada al tratamiento de productos desechables como son el cartón y el plástico; esta actividad contribuye grandemente a reducir la contaminación, ya que cuentan con programas de recolección de estos residuos en las principales empresas, escuelas y universidades de la Región estudiada.

De igual forma, se localizó una sociedad de producción rural que promueve el cuidado y la preservación del medio ambiente, así como la producción con fertilizantes orgánicos para brindar una alimentación sana a sus habitantes. Además promueve el desarrollo sustentable o sostenible que surge como una manera de responder a las exigencias de la comunidad, principalmente en las áreas ecológicas y ambientales así como en las áreas económicas y sociales. Esta misma sociedad tiene como tarea enseñar a los productores a elaborar sus propios biofertilizantes, mediante cursos de capacitación donde interactúan con experiencias de expositores de otros países de Latinoamérica. 
De igual forma, tiene programada dentro de sus actividades la difusión del cuidado del medio ambiente y la alimentación sana, desde los niveles básicos de educación: preescolar, primaria y secundaria, donde se promueve la arborización que va desde la adopción de un árbol hasta la conciencia de cuidar el agua, los animales, y separar la basura. También se invita al consumo de alimentos sanos, involucrando a profesores y madres de familia a que reorienten los alimentos que ofrecen a los niños en las tiendas escolares.

Los microempresarios reconocen que es necesaria la práctica de los principios de RS, como un deber moral para todo tipo de organizaciones, ya que no se establecen en una ley, sino son principios establecidos internacionalmente y que deben ser observados y practicados como una estrategia de negocios que les permite distinguirse en el mercado que participan. Coinciden los empresarios de la Región en que estas prácticas responsables generan costos a la organización, pero que son recuperados en el tiempo; es notable la preocupación de estas organizaciones que han empezado a sensibilizarse y a hacer conciencia con el ambiente y con los recursos humanos que laboran en ellas. Las empresas buscan satisfacer sus necesidades tomando en cuenta el impacto que sus actividades generan. Sin embargo, todavía existen empresas que no pueden implementar este tipo de acciones ya que no cuentan con una estructura completa en su organización.

\section{Principales ventajas que obtienen los microempresarios con la práctica de acciones responsables}

Se pudo apreciar cómo los empresarios están conscientes de que la RSE ayuda a que la empresa sea más competitiva, no solo por sus incrementos en los beneficios económicos, sino también porque juega un papel esencial en el desarrollo positivo de factores vitales para la supervivencia, la sostenibilidad y el éxito de la empresa a largo plazo.

Otra ventaja es que las empresas que están desarrollando prácticas socialmente responsables, refuerzan su imagen externa e institucional y les otorga mayores niveles de credibilidad, lealtad y reconocimiento entre sus clientes, trabajadores, proveedores, autoridades y comunidad en general; además facilita su posicionamiento estratégico en el mercado y la estabilidad en su plantilla de trabajadores.

También los consumidores reconocen el esfuerzo que hacen las microempresas para desempeñarse como empresas socialmente responsables que valoran a sus empleados, participan en proyectos sociales en bien de la comunidad y se preocupan por el medio ambiente, y sobre todo, que luchan contra las prácticas corruptas en sus negocios, por lo tanto, la RS es un factor que garantiza la fidelidad del consumidor. 


\section{Conclusiones}

La RSE se puede definir como un término que hace referencia al conjunto de obligaciones y compromisos, legales y éticos, tanto nacionales como internacionales, que se derivan de los impactos que la actividad de las organizaciones producen en el ámbito social, laboral, medioambiental y de los derechos humanos, asumidos por la gestión de la empresa con el objetivo de mejorar su situación competitiva y agregar valor a sus operaciones. Estas prácticas se enmarcan dentro de los principios y las políticas de RSE.

Por lo tanto, implementar principios y políticas de RS en las microempresas representa grandes beneficios competitivos para su posicionamiento en el mercado; entre los cuales pudimos rescatar los siguientes:

- Genera un ambiente laboral armonioso entre los empleados, ya que al brindar beneficios de previsión social mejora la calidad de vida del trabajador y de sus familias.

- La empresa tiene la oportunidad de construir o reconstruir la organización, tornándola en una entidad más justa y eficiente, que a medida que avanzan en la práctica de los principios y políticas de RS, logra mejorar las condiciones laborales e incrementan su productividad, lo que repercute de manera positiva en sus resultados.

- Contribuye a que la empresa sea distinguida de la competencia al practicar acciones responsables, que le ayudan a atraer nuevos clientes y mayores niveles de credibilidad, lealtad y reconocimiento de los mismos.

- La RSE es esencial en el desarrollo de la microempresa, ya que es una herramienta eficaz para mejorar las relaciones con los clientes, ya que mejora criterios de eficiencia, sostenibilidad e igualdad de oportunidades, el respeto, la tolerancia y la integración de los elementos que componen la empresa.

- Las prácticas socialmente responsables agregan valor a los negocios de la empresa y pueden ser un diferenciador para sus estrategias de negocios.

- Los empresarios encuentran un público preocupado por la ética y el tratamiento a los empleados dispuestos a premiar a empresas que adoptan estos comportamientos éticos.

- La RS garantiza la permanencia del negocio a largo plazo, ya que disminuye los riesgos de la empresa y permite un control más exhaustivo, reduciendo la inseguridad del negocio.

- Encuentran satisfacción en la aportación que se hace a la sostenibilidad con sus buenas prácticas medioambientales. 
La práctica de la RSE es una estrategia comercial y competitiva que puede ser considerada como un activo intangible que agrega valor a la empresa, que fomenta el compromiso de los trabajadores y permite el reconocimiento de consumidores y grupos de interés de las empresas. Queda demostrado que la RSE contribuye eficazmente a que las conductas sociales se tornen más respetuosas en las relaciones interpersonales y se tenga mayor conciencia hacia el cuidado y la preservación del medio ambiente.

\section{Bibliografía}

Abreu, J. L. y Badii, M. (2007). Análisis del concepto de responsabilidad social empresarial. Octubre 2006 - Marzo 2007. Disponible en: http://www.spentamexico. org/v2-n1/2(1)\%2054-70.pdf consultado el 28 de abril del 2014.

Agüero, F. (2002). La Responsabilidad Social Empresarial en América Latina: Argentina, Brasil, Chile, Colombia, México y Perú. Escuela de Estudios Internacionales, Universidad de Miami, Grupo RSE-CHILE.

Andrade, Simón (2005). Diccionario de Economía. Tercera Edición, Editorial Andrade, pág. 448.

Aragón Medina, Jorge y Rocha Sánchez, Fernando (2009). "Los actores de la responsabilidad social empresarial: el caso español". Cuadernos de Relaciones Laborales, Vol. 27, núm. 1, pág. 164. Disponible en: file://C:/ Users/Invitado/Downloads/Art.\%20Responsabilidad\%20Social\%20 de $\% 201$ as $\% 20$ empresas\%20\%202009.pdf consultado el 22 de enero del 2014.

Argandoña, Antonio (2012). Otra definición de responsabilidad social. Comentarios de la cátedra de la "Caixa”. Disponible en: http://www.iese.edu/en/files/ catedra\%20abril_tcm4-79450.pdf consultado el 25 de enero del 2014.

Ávila Baray, Héctor Luis (2006). Introducción a la metodología de la investigación. Disponible en: http://www.eumed.net/libros-gratis/2006c/203/2k.htm consultado el 04 de junio del 2014.

Brown Grossman, Flor (2010). "Los principios de la responsabilidad social empresarial". Economia Informa, núm. 363 marzo-junio 2010. Disponible en: http://www. economia.unam.mx/publicaciones/econinforma/pdfs/363/06florbrown.pdf consultado el 14 de marzo del 2014.

CEMEFI (2012). Concepto de Responsabilidad Social Empresarial. Disponible en http:// www.cemefi.org/esr/images/stories/pdf/esr/concepto_esr.pdf consultado el 18 y el 20 de enero del 2014. 
Comisión de las Comunidades Europeas (2001). Libro verde. Fomentar un marco europeo para la responsabilidad social de las empresas. Disponible en: http:// www.jussemper.org/Inicio/Recursos/Actividad\%20Corporativa/Resources/ Libro\%20verde.pdf consultado el 28 de abril del 2014.

Correa Jaramillo, Juan Guillermo (2007). "Evolución histórica de los conceptos de Responsabilidad Social Empresarial y balance social”. Semestre Económico, núm. julio - diciembre, pp. 87-102. Disponible en: http://www.redalyc.org/ articulo.oa?id=165013675005 consultado el 24 de abril del 2014 .

Dávila Sánchez, Aracely y Gómez Solís, José Luis (2009). "Responsabilidad Social Empresarial: un reto para la industria química”. Synthesis, octubrediciembre 2008. Disponible en: http://www.uach.mx/extension_y_difusion/ synthesis/2009/04/27/Responsabilidad_social_empresarial_un_reto_para_ la_industria_quimica.pdf

Delgado Gallart, Roberto (2013). “QQué implica la Responsabilidad Social Empresarial?”. El Economista. Disponible en: http://eleconomista.com. $\mathrm{mx} /$ columnas/columna-invitada-empresas/2013/09/09/que-implicaresponsabilidad-social-empresarial consultado el 28 de abril del 2014.

Foretica (2014). Qué es la RSE, disponible en: http://www.foretica.org/conocimientorse/que-es-rse?lang=es consultado el 28 de abril del 2014.

Fundación con Responsabilidad Social "FUNDARSE" (2009). Principios generales de la RSE. Disponible en: http://www.fundarse.org/principios-generales-dela-rse.html consultado el 12 de marzo del 2014.

González Rodríguez, Consuelo (2011). "Empresas socialmente responsables y mercado verde internacional". Economía informa, núm. 366, enero-febrero 2011. Disponible en: http://www.economia.unam.mx/publicaciones/ econinforma/pdfs/366/08consuelo.pdf. consultado el 11 de marzo del 2014.

Guzmán Mendoza, José Oscar (2004). La responsabilidad social empresarial. Disponible en: http:www.gestiopolis.com/canales3/ger/ressocemp.htm, consultado el 15 de marzo del 2014.

INEGI (2009). Micro, pequeña, mediana y gran empresa, censos económicos 2009. Disponible en: http://www.inegi.org.mx/est/contenidos/espanol/proyectos/ censos/ce2009/pdf/Mono_Micro_peque_mediana.pdf consultado el 19 de enero del 2014.

ISO 26000 (2010). Guía sobre responsabilidad social. Disponible en: www.iso.org/iso/ iso_26000_projet_overview-es.pdf consultado el 28 de mayo del 2014.

Juholin, E. (2003). Born Again. A Finnish Appoach to Corporate Social Responsibility, University of Jyväskylä, Jyväskylä. 
LDCMPME (2014). Ley para el Desarrollo de la Competitividad de la Micro, Pequeña y Mediana Empresa. Disponible en: http:www.diputados.gob. $\mathrm{mx} /$ LeyesBiblio/ref/ldcmpme.htm consultado el 15 de noviembre de 2014.

Malave, Néstor (2007). Escala tipo Likert. Disponible en: http://uptparia.edu.ve/ documentos/F\%C3\%ADsico\%20de\%20Escala\%20Likert.pdf consultado el 06 de junio del 2014.

Mirell Malfavon, Pamela (2014). Empresa familiar. Disponible en: http://www.esmas. com/emprendedores/pymesint/empresasfiliales/493321.html. consultado el 30 de mayo 2014

Monje Álvarez, Carlos Arturo (2011). Metodología de la investigación cualitativa y cuantitativa. Disponible en: http://carmonje.wikispaces.com/file/view/Mon je+Carlos+Arturo++Gu\%C3\%ADa+did\%C3\%Alctica+Metodolog\% $\% 3 \%$ $\mathrm{ADa}+$ de+la+investigaci\%C3\%B3n.pdf consultado el 08 de junio del 2014.

Moreno Freites, Zahira y Graterol, Dennys (2011). "Prácticas de responsabilidad social empresarial en la Pyme. Un estudio en el sector metalmecánico de Barquisimeto, Estado Lara, Venezuela”. Contaduría y administración. Disponible en: http://www.scielo.org.mx/scielo.php?script=sci_arttext\&pid =S018610422011000300009 consultado el 13 de marzo del 2014 .

Núnez, Georgina (2006). Responsabilidad social empresarial - CEPAL Washington. Disponible en: http://www.eclac.org/cgibin/getProd.asp?xml=/cooperacion/ noticias/noticias/6/26446/P26446.xml\&xsl=/cooperacion/tpl/p1f. xsl\&base=/cooperacion/tpl/topbottom.xslt consultado el 18 de enero del 2014.

Ortiz Arrieta, Luis Bonilla (2011). "Evolución del concepto de Responsabilidad Social Empresarial (RSE)”. El observador económico. Una publicación de la Fundación, consultado el 02 de mayo del 2014.

Organización Internacional del Trabajo - OIT (2010). "La OIT y la responsabilidad social de la empresa (RSE)”. Helpdesk de la OIT, núm. 1. Disponible en: http://www.ilo.int/wcmsp5/groups/public/---ed_emp/---emp_ent/---multi/ documents/publication/wcms_142694.pdf. y http://www.ilo.int/empent/ Publications/WCMS_142694/lang--es/index.htm. consultado el 13 de marzo del 2014.

Osorio Rojas, Ricardo Arturo (2012). El cuestionario. Disponible en: http://www. nodo50.org/sindpitagoras/Likert.htm consultado el 08 de junio del 2014.

Pacto Mundial (2014). Principios de Responsabilidad Social Empresaria. Disponible en: http://www.unglobalcompact.org/languages/spanish/los_diez_principios. html consultado el 30 de mayo del 2014. 
Sanborn, C. (2004). La filantropia "realmente existente" en América Latina. Colombia, Departamente de Ciencias Sociales y Políticas, Centro de Investigación de la Universidad del Pacífico, Bogotá.

Sánchez, M. Carol (2000). "Motives for corporate philantropy in El Salvador. Altruism and political legitimacy. Journal for Business Ethics.

Segura Cáceres, Ana Graciela (2012). Responsabilidad social “Artículo ampliado”. Disponible en: http://blogs.cnnexpansion.com/soy-competitivo/2012/07/15/ responsabilidad-social-articulo-ampliado/ consultado el 15 de marzo del 2014.

\section{Cómo citar este artículo:}

González, M. C. y López, M. L. (2014). “Responsabilidad Social desde la microempresa comercial”, Oikos No 37, 105 - 126, Escuela de Administración y Economía, Universidad Católica Silva Henríquez (UCSH), Santiago de Chile. [http://ediciones.ucsh.cl/revistas.php]

Fecha de recepción: 03 / $11 / 2014$

Fecha de aceptación: 08 / 12 / 2014 